\title{
The Python's Embrace: Clinical Research Regulation by Institutional Review Boards
}

Subject consent and its waiver are critical topics in contemporary research. Institutional review boards (IRBs), acting under the wary eye of the Office for Human Research Protections (OHRP), typically may waive consent when research involves no more than minimal risk, waiver would not harm the subjects, and the research would be impracticable without the waiver. ${ }^{1}$ IRBs constrict research when they cautiously interpret "impracticable" to mean "impossible" and insist on consent. And, in an ironic twist, IRBs can thus injure the vulnerable groups they imagine they are protecting.

A disturbing pair of articles by Rich and his colleagues, one from $2010^{2}$ and the second in this issue of Pediatrics, ${ }^{3}$ demonstrate how this works. SUPPORT, the parent study, investigated the impact of different respiratory care regimens on lung disease, retinopathy, and survival in premature infants born between 24 and 27 weeks' gestational age. ${ }^{4,5}$ Eighteen IRBs reviewed the protocol; all required that consent be obtained, even though these interventions are routinely provided without specific consent in everyday practice. This study reveals 3 of the resultant costs.

The first cost is time and money. Obtaining consent drained $\$ 200000$ from the project, and if $75 \%$ of the eligible but not enrolled infants had been included, recruitment would have been completed in 18 instead of 48 months. The requirement for informed consent also led to the second, predictable ${ }^{6}$ cost of biased trial entry. Compared with mothers of infants enrolled in the trial, mothers of infants not enrolled were less likely to be white, insured, have a high school education, and have received prenatal care. As the article in this issue demonstrates, ${ }^{3}$ their infants were also more likely to die.

African American infant mortality in the United States is double that of white infants. ${ }^{7}$ The IRB system's stated goals include the pursuit of justice and the protection of vulnerable groups; yet the SUPPORT findingsfruits of the hard work of physicians and nurses at 18 major medical centers over 4 years-are, as a result of IRB action, more applicable to white infants than to black infants.

The IRB system's third cost, and its greatest error, is hidden in plain sight: patients die while better treatments are still in development. During the years SUPPORT was delayed, neonatologists, awaiting the trial findings, knew less about how to save infants in their care from respiratory failure, blindness, and death. Collins et al ${ }^{8}$ first articulated the fundamental issue at work here-that when regulation delays lifesaving research, lives are lost. Schneider and I have confirmed the estimate of Collins et al that consent-related delays in research in heart attack treatment in the 1980s cost thousands of lives. ${ }^{9}$ Beauchamp ${ }^{10}$ recently made a similar point, noting that OHRP's delay of the Keystone initiative to reduce ICU infections, if continued, might have caused

\section{AUTHOR: Simon N. Whitney, MD, JD}

Department of Family and Community Medicine, Baylor College of Medicine, Houston, Texas

The author is entirely responsible for this submission. Dr Whitney received suggestions from the individuals identified in the acknowledgments, but none meet the criteria for authorship.

Opinions expressed in these commentaries are those of the author and not necessarily those of the American Academy of Pediatrics or its Committees.

www.pediatrics.org/cgi/doi/10.1542/peds.2011-3455

doi:10.1542/peds.2011-3455

Accepted for publication Nov 21, 2011

Address correspondence to Simon N. Whitney, MD, JD, Department of Family and Community Medicine, Baylor College of Medicine, 3701 Kirby Dr, Suite 600, Houston, TX 77098. E-mail: swhitney@bcm.edu

PEDIATRICS (ISSN Numbers: Print, 0031-4005; Online, 1098-4275) Copyright (C) 2012 by the American Academy of Pediatrics FINANCIAL DISCLOSURE: The author has indicated he has no financial relationships relevant to this article to disclose.

FUNDING: Dr Whitney is supported in part by the William W. O'Donnell, MD, \& Regina O'Donnell Chair in Family Medicine at Baylor College of Medicine and by the Center for Clinical Research and Evidence-Based Medicine at the University of Texas Health Science Center at Houston Medical School, Houston, Texas.

COMPANION PAPER: A companion to this article can be found on page 480 and online at www.pediatrics.org/cgi/doi/10.1542/ peds.2011-2121 
unnecessary patient deaths. To Dingwall, ${ }^{11}$ a British sociologist, 1 cost of ethics constriction of his study of why physicians and nurses reuse singleuse medical devices is "the lives that will not be saved." And, Heimer has said, "The biggest ethical lapse in American regulation of human subjects research is the death and suffering that has resulted from slowing the pace and altering the focus of research and squandering research funds." 12 These are serious charges, but no defender of the system has argued that Collins, Beauchamp, Dingwall, Heimer, or Schneider and I are mistaken. Just as a python's victim shows no outward signs of trauma, biomedical protocols appear little changed by regulatory constriction. But IRB regulation of clinical research costs lives as surely as the python's embrace. We can intelligently discuss a system of social control only if we understand its nature. This system, comprising OHRP and the IRBs it regulates, functions as a federal regulatory agency. Its workers are employed by a variety of federal, state, and private institutions, but federal regulations created IRBs, a federal office oversees them, and IRBs refer to federal law to justify their actions. Like any governmental invention, this system's merits and defects should be scrutinized and its value vigorously debated. Although its defenders invoke ethics to justify its existence and impeach its critics, ${ }^{13-15}$ this system is not intrinsically moral, nor is its existence mandated by past abuses. No special shield protects it from folly or error.

Thirty years ago, Levine ${ }^{16}$ estimated that IRBs cost $\$ 130000000$ per year, and asked the right question: "What do we think we are buying with all this money?" The answer requires the same analysis we would make of any other agency. A legitimate regulatory system produces societal benefits that exceed its costs. Ethicists have left the costs and benefits of the IRB system untouched, but this vacuum has begun to be filled by experts in law and public policy. At a symposium at Northwestern University School of Law in 2007, Hyman ${ }^{17}$ stated that "there is no empirical evidence that IRBs have any benefit whatsoever," and Zywicki ${ }^{18}$ concluded that the system's costs "substantially exceed" its benefits.

No proponent of this regulatory regimen has stepped forward to rebutthese charges. Instead, there are calls for reform, ${ }^{19,20}$ including proposals by the federal government itself. ${ }^{21}$ But if Hyman ${ }^{17}$ and Zywicki ${ }^{18}$ are correct, the logical response to the system's failings is not reform. The logical response is abolition.

\section{ACKNOWLEDGMENTS}

The author acknowledges the insightful comments of Karen Brisch, Mats Hansson, Kathleen Kennedy, Paula Knudson, Patricia Serventi Naughton, Heidi Russell, Carl E. Schneider, Jon Tyson, and Susan Wootton.

\section{REFERENCES}

1. 45 CFR 46.116(d)

2. Rich WD, Auten KJ, Gantz MG, et al; National Institute of Child Health and Human Development Neonatal Research Network. Antenatal consent in the SUPPORT trial: challenges, costs, and representative enrollment. Pediatrics. 2010;126(1). Available at: www.pediatrics. org/cgi/content/full/126/1/e215

3. Rich W, Finer NN, Gantz MG, et al; SUPPORT and Generic Database Subcommittees of the Eunice Kennedy Shriver National Institute of Child Health and Human Development Neonatal Research Network. Enrollment of extremely low birth weight infants in a clinical research study may not be representative. Pediatrics. 2012;129(3):480-484

4. Finer NN, Carlo WA, Walsh MC, et al; SUPPORT Study Group of the Eunice Kennedy Shriver NICHD Neonatal Research Network. Early CPAP versus surfactant in extremely preterm infants. N Engl J Med. 2010;362 (21):1970-1979

5. Carlo WA, Finer NN, Walsh MC, et al; SUPPORT Study Group of the Eunice Kennedy Shriver NICHD Neonatal Research Network.

Target ranges of oxygen saturation in extremely preterm infants. $N$ Engl J Med. 2010;362(21):1959-1969

6 . Tyson J. Dubious distinctions between research and clinical practice using experimental therapies: have patients been well served? In: Goldworth A, ed. Ethics and Perinatology. New York, NY: Oxford University Press; 1995:214-230

7. Mathews TJ, MacDorman MF. Infant mortality statistics from the 2007 period linked birth/infant death data set. Natl Vital Stat Rep. 2011;59(6):1-30

8. Collins R, Doll R, Peto R. Ethics of clinical trials. In: Williams CJ, ed. Introducing New Treatments for Cancer: Practical, Ethical and Legal Problems. Chichester, UK: John Wiley \& Sons Ltd; 1992:49-65

9. Whitney SN, Schneider CE. Viewpoint: a method to estimate the cost in lives of ethics board review of biomedical research. J Intern Med. 2011;269(4):396402

10. Beauchamp TL. Viewpoint: why our conceptions of research and practice may not serve the best interest of patients and subjects. J Intern Med. 2011;269(4):383387

11. Dingwall R. The ethical case against ethical regulation in humanities and social science research. 21st Century Society. 2008;3(1):1-12

12. Heimer CA. The unstable alliance of law and morality. In: Hitlin S, Vaisey S, eds. Handbook of the Sociology of Morality. New York NY: Springer Science+Business Media; 2010:179-202

13. Feeley MM. Response to comments. Law Soc Rev. 2007;41(4):811-818

14. Burris S. Regulatory innovation in the governance of human subjects research: a cautionary tale and some modest proposals. Regulation \& Governance. 2008;2(1):65-84

15. van den Hoonaard WC. The Seduction of Ethics: Transforming the Social Sciences Toronto, Canada: University of Toronto Press; 2011:103-110

16. Levine RJ. Ethics and Regulation of Clinical Research. Baltimore, MD: Urban \& Schwarzenberg; 1981:241 
17. Hyman D. Institutional review boards: is this the least worst we can do? Northwestern University Law Review. 2007;101 (2): :749-774

18. Zywicki TJ. Institutional review boards as academic bureaucracies: an economic and experiential analysis. Northwestern University Law Review. 2007;101(2):861-896
19. Fost N, Levine RJ. The dysregulation of human subjects research. JAMA. 2007;298 (18):2196-2198

20. Emanuel EJ, Menikoff J. Reforming the regulations governing research with human subjects. N Engl J Med. 2011;365(12):1145-1150

21. Department of Health and Human Services, Office of the Secretary, and Food and Drug
Administration. Advanced Notice of Proposed Rulemaking. Human Subjects Research Protections: enhancing protections for research subjects and reducing burden, delay, and ambiguity for investigators (proposed amending 45 CFR Parts 46, 160, and 164 and 21 CFR Parts 50 and 56). Fed Regist. 2011;76(143):44512-44531

DOES ICE DESERVE THE COLD SHOULDER?: Last week, my son pulled a muscle while playing soccer. He immediately applied ice to the skin over the muscle. When he got home he noted that his muscle did not hurt much but that the entire area around the pulled muscle was completely numb and walking felt odd. Within a week, he was playing soccer again but his actions made me wonder whether there is any data to support the use of ice for the treatment of athletic musculoskeletal injuries. According to an article in The New York Times (Blogs: January 4, 2012), despite the widespread belief that ice is beneficial, little data exists to support it. A review of the topic in 2004 concluded that icing decreased pain but could not find strong evidence for any other benefit. Since then, a small randomized trial did not find any benefit to icing muscle injuries. Injured muscles that were iced did not heal faster and weren't less painful than injured muscles with no ice applied. More recently, a review of more than 30 studies concluded that icing helped decrease the pain associated with muscle injury by numbing the area. However, icing was also associated with reduced muscular strength and power, impaired fine motor coordination, and even occasionally impaired proprioception. Most of these effects were short lived, e.g. 15 minutes or so after the ice was removed. Interestingly, athletes who immediately returned to competition after icing, found it detrimental to their athletic performance. Athletes who iced for 20 minutes could not run as fast, jump as high, or throw a ball as well. Whether icing actually leads to an increased risk of injury in athletes who rapidly return to competition was not examined. Why athletes have impaired performance after icing is unknown. Possible explanations include reduced nerve conduction velocity or altered muscle-tendon mechanics. So what are the implications for the average weekend warrior? For those who exercise and experience muscular skeletal pain afterwards, ice is an inexpensive and effective way to decrease pain. For those highly competitive athletes who are seeking to return to play, waiting several minutes after the ice has been removed may be prudent. 